\title{
Minimizing Cost Flow for Colorectal Surgery in Michigan
}

Arjun P. Meka, BS ${ }^{1}$; Todd A. Jaffe, BBA ${ }^{1}$; Daniel Z. Semaan, BS ${ }^{1}$; Uchenna Okoro, BS ${ }^{1}$; Charles Hwang, BS ${ }^{1}$; Joseph Papin IV, BS ${ }^{1}$; Andrew Mullard, MSc'; Michael J. Englesbe, MD ${ }^{1,3}$; Darrell A. Campbell, MD ${ }^{3}$

\section{Introduction}

$\mathbf{S}$ urgical site infections (SSI) are a known cause of increased morbidity and cost following colectomy. ${ }^{1-3}$ Recent work has shown that 6 perioperative care measures are associated with SSI in colectomy patients: (1) Surgical Care Improvement Project-2 antibiotics, (2) postoperative normothermia, (3) oral antibiotics with bowel preparation, (4) perioperative glycemic control, (5) minimally invasive surgery, and (6) short operative duration. ${ }^{6,7}$ The presence of these criteria individually was associated with reduced rates of SSI following colectomy. ${ }^{6-7}$ Given the association between SSI and cost, we hypothesized that when measured together, these perioperative care elements would be predictive of cost per patient. We conducted a retrospective study using data from the Michigan Surgical Quality Collaborative (MSQC) to understand the cost per patient whose care was considered low compliance in these elements $(0-2$ elements) versus those with highcompliance care (3-6 elements).

\section{Methods}

Using MSQC data, which are collected from 73 participating hospitals in the state of Michigan, we identified adult patients $(\geq 18$ years of age) undergoing elective colectomies from fiscal year 2012 to fiscal year 2014. We then refined our study population to those patients who had associated Blue Cross Blue Shield of Michigan (BCBSM) episodic payment data at the patient level.

The selected population was stratified into 2 groups based on compliance with the 6 care measures listed above. A patient's care was considered to be high compliance if he or she had 3 to 6 elements and low compliance if he or she had 0 to 2.

A2-stage approach was used to adjust BCBSM episodic payments for variation in payment attributable to case mix and hospital-level contractual differences. Stage 1 
used a method for claims price standardization developed by ArborMetrix, and stage 2 incorporated for case mix and hospital-level clustering.

\section{Results}

We identified a total of 3387 adult patients undergoing colectomy in the MSQC data set. Of these, 332 (9.8\%) had BCBSM episodic data and were included in our analysis. Of these 332 patients, 33 (9.9\%) had only 0 to 2 care elements ("Iow compliance"), while 299 (90.1\%) had 3 to 6 care elements ("high compliance"). Total episodic payment data were collected across both stratifications (Figure 1). Low compliance with perioperative care had an average episodic cost of $\$ 20$ 952, while high compliance had a total episodic cost of $\$ 15490$. This showed a $\$ 5462$ reduction in cost with high compliance cases $(P<0.01)$.

Total episodic payments were divided into multiple categories: total facility base payment, total professional payment, and its subcategories (prior 30-day spending, surgical procedure, base payment, and total post discharge).

In moving from high-compliance to lowcompliance cases, the total episodic costs decreased by 26.1\% (\$20 952; $\$ 15490)$, the total facility base payments decreased by $20.4 \%$ (\$14 960; \$11 899), and the total professional

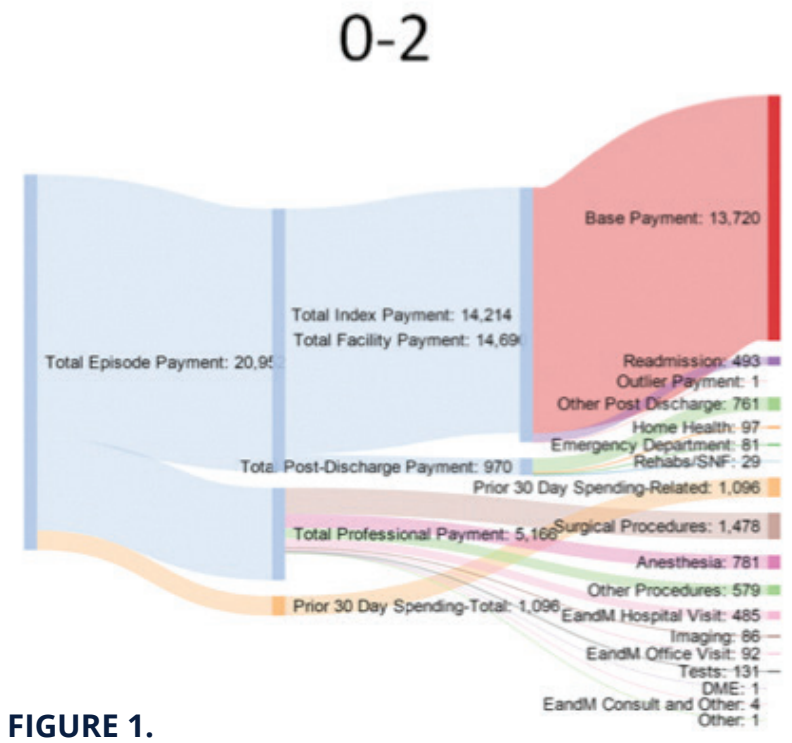

payments decreased by $42.7 \%$ (\$5166; \$2956). The subcategories also showed a trend toward decreased cost for higher bundled care. Prior 30day spending decreased by 42.1\% (\$1096; \$635), surgical procedure spending decreased by $58.3 \%$ (\$1478; \$616), base payment decreased by $16.9 \%$ (\$13 720; \$11 400), and total postdischarge payment decreased by 49.8\% (\$970; \$487). Additional subcategories also showed a decrease in cost trend with higher compliance (Figure 1).

\section{Conclusion}

This retrospective analysis of colectomy patients demonstrates that high compliance with at least 3 of 6 perioperative care elements is associated with decreased cost following elective colectomy in adults with private insurance. One possibility for this cost difference may be SSI, as the 6 care measures have previously been shown to be associated with SSI, ${ }^{6-7}$ and SSI increases costs following colectomy. ${ }^{1-3}$ However, further work is necessary to investigate the pathway between these perioperative care elements and the ultimate cost per patient.

We recognize that the study should be interpreted with limitations. Our study population size was only 332 patients who had private health insurance. Due to our small sample, we were unable

\section{3-6}

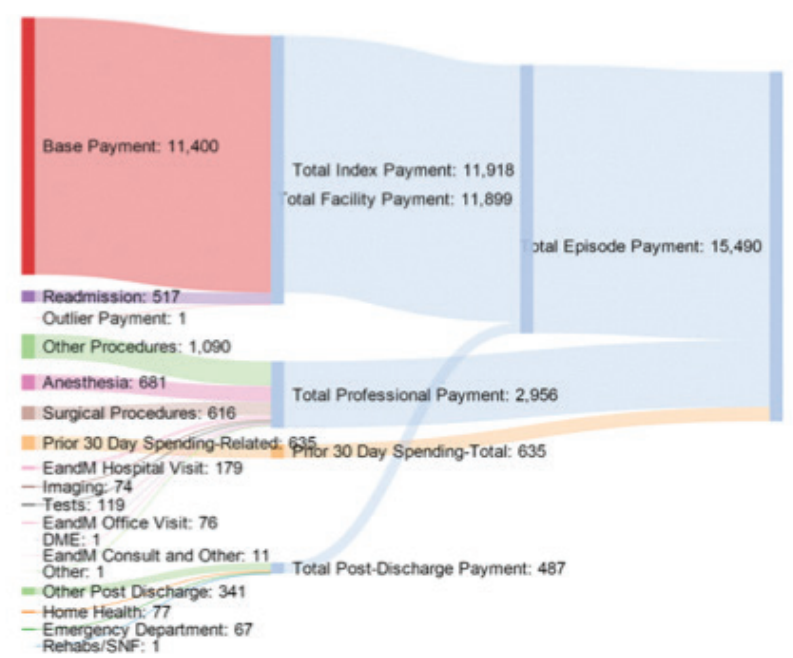

MICHIGAN JOURNAL OF MEDICINE $2016 \cdot 13$ 
to perform a statistical analysis of the cost breakdown in paragraph 3 of the results. In addition, more than $90 \%$ of our study population fell into the "high-compliance" group, limiting our sample of those patients who received poorly compliant care. By selecting a smaller number of patients with the most robust cost data, we were able to study a smaller but more reliable cohort of colectomy

\section{References}

1. National Nosocomial Infections Surveillance System. National Nosocomial Infections Surveillance (NNIS) system report, data summary from January 1992 through June 2004, issued October 2004. Am J Infect Control. 2004;32(8):470-485.

2. Smith RL, Bohl JK, McElearney ST, et al. Wound infection after elective colorectal resection. Ann Surg. 2004;239(5):599-605; discussion 605-597.

3. Wick EC, Vogel JD, Church JM, Remzi F, Fazio VW. Surgical site infections in a "high outlier" institution: are colorectal surgeons to blame? Dis Colon Rectum. 2009;52(3):374-379.

4. Campbell DA Jr., Englesbe MJ, Kubus JJ, et al. Accelerating the pace of surgical quality improvement: patients. This work provides initial data to suggest that compliance with standardized perioperative care elements may be associated with cost. Future work includes understanding cost associations in those populations with an increased risk of complications from less compliance. It is also important to have larger and more diverse populations to further our understanding of the relationships presented.

the power of hospital collaboration. Arch Surg. 2010;145(10):985-991.

5. Campbell DA Jr., Kubus JJ, Henke PK, Hutton M, Englesbe MJ. The Michigan Surgical Quality Collaborative: a legacy of Shukri Khuri. Am J Surg. 2009;198(5 suppl):S49-55.

6. Hendren S, Fritze D, Banerjee M, et al. Antibiotic choice is independently associated with risk of surgical site infection after colectomy: a populationbased cohort study. Ann Surg. 2013;257(3):469-475.

7. Waits SA, Fritze D, Banerjee $M$, et al. Developing an argument for bundled interventions to reduce surgical site infection in colorectal surgery. Surgery. 2014;155(4):602-606. 